\title{
Philosophiques
}

\section{Le sujet du souci : à propos de l'Histoire de la sexualité de Michel Foucault}

\section{Georges Leroux}

Volume 14, numéro 1, printemps 1987

URI : https://id.erudit.org/iderudit/027001ar

DOI : https://doi.org/10.7202/027001ar

Aller au sommaire du numéro

Éditeur(s)

Société de philosophie du Québec

ISSN

0316-2923 (imprimé)

1492-1391 (numérique)

Découvrir la revue

Citer cet article

Leroux, G. (1987). Le sujet du souci : à propos de l'Histoire de la sexualité de Michel Foucault. Philosophiques, 14(1), 5-32. https://doi.org/10.7202/027001ar
Résumé de l'article

Après avoir examiné le chemin parcouru entre La volonté de savoir (1976) et les essais sur le monde antique de 1984, l'A. pose la question du destin du sujet dans une histoire de la sexualité. Comment doit être interprétée, dans les derniers ouvrages de Foucault, la restauration du sujet, sinon comme la rencontre avec le sujet de la philosophie grecque ? L'A. discute ensuite l'identification d'une histoire de la sexualité avec une histoire de la philosophie. 
PHILOSOPHIQUES, Vol. XIV, Numéro 1, Printemps 1987

\title{
LE SUJET DU SOUCI : A PROPOS DE L'HISTOIRE DE LA SEXUALITÉ DE MICHEL FOUCAULT
}

\author{
par Georges Leroux
}

RÉSUMÉ. Après avoir exarniné le chemin parcouru entre La volonté de savoir (1976) et les essais sur le monde antique de 1984, l'A. pose la question du destin du sujet dans une histoire de la sexualité. Comment doit être interprétée, dans les derniers ouvrages de Foucault, la restauration du sujet, sinon comme la rencontre avec le sujet de la philosophie grecque? L'A. discute ensuite l'identification d'une histoire de la sexualité avec une histoire de la philosophie.

ABSTRACT. After an examination of the distance separating $\mathrm{La}$ volonté de savoir (1976) and the essays on the ancient world (1984), this paper raises the question of the destiny of the subject in the framework of a history of sexuality. How should we interpret, in these last works of Foucault, the restoration of the subject, if not as an encounter with the subject of greek philosophy? The paper discusses the identification of a history of sexuality with the history of philosophy.

«... je veux que tu n'aies jamais manqué d'allégresse. Je veux qu'elle foisonne en ton logis. Elle foisonnera, à condition d'être au-dedans de toi-même. (...) tourne ton regard vers le bien véritable; sois heureux de ton propre fonds. Mais ce fonds quel est-il ? Toi-même, et la meilleure partie de toi.»

Sénèque, Lettres à Lucilius, 23, 3 et 6 [Traduction H. Noblot] «... comme le sculpteur, enlève le superflu, redresse ce qui est oblique, nettoie ce qui est sombre pour le rendre brillant, et ne cesse pas de sculpter ta propre statue, jusqu'à ce que l'éclat divin de la vertu se manifeste, jusqu'à ce que tu voies la tempérance siégeant sur un 
trône sacré. Es-tu devenu cela? Est-ce que tu vois cela? Est-ce que tu as avec toi-même un commerce pur, sans aucun obstacle à ton unification, sans que rien d'autre soit mélangé intérieurement avec toi-même?»

Plotin, Ennéade I,6,9, 13-17

[Traduction E. Bréhier]

Dans un petit livre paru juste après La volonté de savoir, Jean Baudrillard proposait d'oublier Foucault. L'attaque était brutale, incisive, mortifiante. Surveiller et punir était qualifié de magistral, mais révolu. Plus, le programme entier de l'histoire de la sexualité, tel que Foucault l'exposait dans La volonté de savoir, se trouvait récusé par avance, pour des motifs dont l'importance peut à nouveau être discutée, maintenant que nous pouvons lire les deux premiers volumes de cette histoire de la sexualité. Ces études sur la sexualité dans la culture et la pensée gréco-romaines sont parues à l'été de 1984, huit ans après le premier énoncé des hypothèses de recherche et quelques semaines avant la mort de Michel Foucault.

L'objection de fond, et Baudrillard n'en était en son temps que l'écho porteur, peut être abordée de manière simple. Dans l'entreprise de constitution d'une histoire de la sexualité, où se conjuguent un travail de construction et un travail d'interprétation, il ne suffit pas de chercher à substituer une conception du pouvoir à une autre. Peut-on se satisfaire que Foucault tente de mettre en place la positivité d'un pouvoir actif et immanent, destinée à contrer une conception négative et réactionnelle? 1 Que signifie l'introduction du dispositif au lieu et à la place de l'autorité, si on ne peut encore rendre compte de la dissolution du sujet? Comment expliquer, enfin, que l'édifice ainsi construit n'intègre aucun concept de désir? Cet ensemble de questions ne trouve pas facilement réponse dans La volonté de savoir. La lecture du programme de 1976 montre en effet une hypothèse complexe, hostile non seulement aux concepts de la psychanalyse, mais à l'ensemble des concepts constitutifs d'une théorie du sujet

1. La question anime tout l'essai de Jean Baudrillard. Oublier Foucault. Paris, Éditions Galilée, 1977. 
et principalement attentive au régime des effets de pouvoir et de savoir qui soutient le discours sur la sexualité humaine. Dans ce programme donc, la place du sujet, la place du désir est déjà prise. Tout est occupé, envahi par cette micro-physique du pouvoir dont le système explicatif s'applique aussi bien à l'histoire de l'enfermement qu'à l'histoire de la sexualité.

Le cas particulier de l'histoire de la sexualité a été l'occasion, dans un premier moment, de l'élaboration méthodique d'un dispositif dont Foucault avait déjà annoncé le schéma général dans sa contribution au numéro de Critique (1963) consacré à Georges Bataille, intitulée «Préface à la transgression " ${ }^{2}$. Le sexe est d'abord discours. La sexualité a été conduite aux limites de la conscience, devenant en quelque sorte la matrice de la seule interprétation plausible de l'inconscient; elle a été conduite aux limites du langage, marquant les limites de la conquête du silence. Dans cette instanciation par le discours se lit l'essentiel de l'appareil de La volonté de savoir. A cet appareil font défaut la séduction et la gratuité, la générosité des rituels avec leurs dons et contre-dons, et tout ce qui, pour Baudrillard et d'autres encore, constitue le désir autrement que dans ses formes de pouvoir. Il y manque certes le sujet, centre de gravité, mais encore cet autre sujet, disséminé et dispersé dans le jeu des simulacres et le destin naturel des pulsions. Baudrillard ne veut pas postuler contre Foucault l'avènement d'un sujet sexuel, susceptible de fournir une cohérence à une histoire de la sexualité. "La cohérence et la transparence de l'bomo sexualis n'a jamais eu plus de réalité que celle de l'bomo oeconomicus. " ${ }^{3}$ Même la psychanalyse doit être mise en cause, et sur ce point Baudrillard rejoint Foucault : faire l'économie des topiques et des instances d'un sujet dramatisé, structuré, mis en scène.

Cela même en effet, Foucault l'avait amorcé avec grande sévérité dans La volonté de savoir. La proposition principale de cet ouvrage programmatique était de n'accorder aucun statut à une hypothèse de refoulement, aucune originalité à la répression.

2. Michel Foucault. «Une Préface à la transgression». Critique, nos 195-196 (1963) p. 751.

3. Jean BAUdRIlLARD, op. cit., p. 39. 
Le sexe n'est pas d'abord refoulé ou réprimé, mais parlé. Tous les éléments négatifs, "défenses, refus, censures, dénégations que l'hypothèse répressive regroupe en un grand mécanisme central destiné à dire non, ne sont sans doute que des pièces qui ont un rôle local et tactique à jouer dans une mise en discours, dans une technique de pouvoir, dans une volonté de savoir qui sont loin de se réduire à eux. ${ }^{4}$ La constitution progressive de ce discours, de cette technique, de cette volonté est à proprement parler la constitution du sujet, qui apparaît comme son produit. Le sujet, pour autant qu'on puisse ici parvenir à le représenter, est la résultante de sa causalité propre, de son inconscient, de sa vérité dans l'autre, tous objets du discours du sexes. Ce discours n'est lui-même que l'enveloppe d'un pouvoir protéique, omniprésent.

La critique de Baudrillard ne s'appuyait sur aucun concept particulier du sujet. Elle en avait bien plutôt au caractère, finalement transcendantal et formel, de toutes ces instanciations du pouvoir qui en cherchant à supplanter le sujet finissent par en adopter la position, théorique, spéculative, dramatique. Le pouvoir, chez Foucault, est actif et structurant; il pénètre et détermine, il contrôle et effectue. Dans le concept de stratégie, qui semble le plus adéquat pour représenter sa singulière efficacité, le sujet est à la fois dissous et restitué. Dissous, parce que le nom propre est barré, le centre dispersé ; mais restitué, dans la mesure où une causalité fonctionnelle est rétablie. Stratifiée, sédimentée, certes, mais explicative et efficace. Ce paradoxe rend trouble jusqu'à la méthode de La volonté de savoir, puisqu'il met en question la praticabilité d'une entreprise sans le recours aux concepts de vérité qu'elle se donne pour objectif d'écarter ${ }^{6}$.

Notre étonnement est donc grand, assez considérable pour qu'il soit prévu par Foucault lui-même, quand, à la lecture des tomes suivants de l'histoire de la sexualité, on ne voit rien

4. Michel Foucault. La volonté de savoir. Paris, Gallimard, 1976. (Bibliothèque des Histoires), p. 21

5. Ibid., pp. 93-94.

6. Voir les remarques critiques de Charles TAYLOR. «Foucault on Freedom and Truth. » Political Theory 12, 2 (1934) 152-183. Par exemple, quand il écrit : «... purposefulness without purpose requires a certain kind of explanation to be intelligible». (p. 169) 
réapparaître de la problématique du pouvoir. Tout l'appareil de La volonté de savoir a reflué, il est littéralement refoulé, et à sa place le sujet fait retour. Il fait retour sous la figure du sujet moral de la philosophie grecque classique, et notamment du sujet platonicien dualiste. Ce sujet n'est porté par aucun pouvoir, mais il est pouvoir de lui-même et sur lui-même. Il n'est l'effet d'aucun discours, mais sujet de la philosophie, maître de sagesse, auteur souverain de sa propre existence.

Quel parcours a conduit Foucault de l'analytique du pouvoir, esquissée à l'écart d'une théorie du sujet, à une histoire de la sexualité qui se présente d'emblée comme la restauration du sujet? Cette première question en amène immédiatement une autre : comment une histoire de la sexualité est aussi et en même temps une histoire de la philosophie? La suite de notre essai est un effort pour faire travailler ces deux questions.

\section{I}

Dans la perspective nouvelle qui se dégageait en 1976 avec La volonté de savoir, qu'on peut commodément résumer par le projet de substituer une généalogie à l'archéologie qui avait animé les travaux précédents, il n'y avait guère de place pour une motivation psychologique des morales et des institutions. Foucault parle de stratégies sans stratèges. La métaphorie qui s'empare alors de son vocabulaire possède des visées descriptives, dont la portée principale est l'exclusion d'une causalité forte du sujet. Ni la vérité, ni le pouvoir lui-même ne sont le produit des motivations du sujet ${ }^{7}$. Ce sont, si on s'efforce de démonter le vocabulaire de la stratégie, des organisations sans maître. « ... vous verrez, écrit Foucault, que les effets de domination du pouvoir ne sont pas attribués à une appropriation, mais à des dispositions, à des manœuvres, à des tactiques, à des techniques, à des fonctionnements... ${ }^{8}$ ” La causalité qui se trouve ainsi écartée est à la fois une causalité de contrôle et une causalité de direction; la domination autant que la finalité sont barrées, nous

7. Michel Foucault. Surveiller et punir. Paris, Gallimard, 1975 (Bibliothèque des histoires); p. 31.

8. Ibidem. 
abandonnant à une forme singulière de la subjectivité vide, proche de la volonté schopenhauerienne?. «Nul n'est responsable d'une émergence; nul ne peut s'en faire gloire; elle se produit toujours dans l'interstice. ${ }^{10}$ ”

La généalogie est le savoir de l'interstice. Cette formule se referme sur celui qui l'adopte, elle le prive d'un lieu d'où il pourrait considérer l'avènement du sujet; il n'y a pas de lieu en effet qui, hors discours, ouvrirait l'espace d'un sujet constituant, mais seulement un ensemble de fentes et de fissures dont cherche à rendre compte cette forme d'histoire particulière qu'est la généalogie. Sa tâche première est de se débarrasser du sujet constituant, du sujet lui-même, pour le reconstituer ensuite "dans la trame historique ${ }^{11}$.»

Comment le sujet est dans la trame historique sans être le sujet constituant, il n'est pas facile de l'expliquer. Ce paradoxe vient en effet à bout du contenu du sujet, de sa causalité propre, en quoi résidait sans doute l'essentiel de son concept dans la philosophie classique, et en dispose comme d'une fiction à analyser. Il y a donc, d'une part, le sujet qui est illusion pour luimême et pour une histoire qui s'élabore dans le profil de sa maîtrise, et, d'autre part, le sujet objectivé, produit d'une histoire qui se construit sans lui. Même si Foucault parle encore du sujet au moment où il met en place les procédures destinées à l'effacer, il ne faut peut-être voir là que la volonté de passer d'un sujet à un autre. Du sujet souverain et constituant au sujet-effet produit par et dans l'interstice. L'histoire est savoir de cet interstice, généalogie des productions discursives, des effets de pouvoir et de la volonté de savoir.

Dans le cas particulier de l'histoire de la sexualité, la production discursive prend d'abord la figure d'une incitation

9. J'emprunte ce rapprochement à Charles TAYLOR, op. cit., p. 170

10. Michel Foucault. «Nietzsche, la généalogie, l'histoire». Hommage à Jean Hyppolite. Paris, Presses Universitaires de France, 1971 ; p. 156.

11. Michel Foucault. «Vérité et pouvoir. Entretien avec M. Fontana. La crise dans la tête. » $L^{\prime} A r c, \mathrm{n}^{\circ} 70$ (1977) p. 20 : «Et c'est ce que j'appellerais la généalogie, c'est-àdire une forme d'histoire qui rende compte de la constitution des savoirs, des discours, des domaines d'objets etc., sans avoir à se référer à un sujet qu'il soit transcendant par rapport au champ d'événements ou qu'il courre dans son identité vide, tout au long de l'histoire." 
croissante à la mise en discours du sexe. Le pouvoir, quant à lui, infiltre en se disséminant tous les domaines de la vie sexuelle. Il s'inscrit dans tout l'espace de jeu qui règle méticuleusement les formes de son emprise par la technologie politique du corps. La forme principale de ce rituel est l'aveu, qui est à la fois rituel d'expression et rituel d'attention. Dans l'aveu, dans la confession chrétienne par exemple, la vie sexuelle est, d'une part, nommée et communiquée, d'autre part soumise à l'examen. Ce centrement sur l'aveu va de pair chez Foucault avec l'insistance sur la mise en discours du sexe, avec le questionnement sur la formation de ce discours dont la prolifération moderne lui apparaît constitutive de notre dispositif de sexualité. Cette mise en discours renforce à son tour la volonté de savoir qui produit la scientia sexualis de notre temps; non pas d'abord la psychanalyse, mais l'ensemble des avatars de la médecine psychiatrique. C'est ce triple ensemble de l'incitation au discours, qui prend surtout corps dans l'aveu chrétien de la chair, mais ne s'y limite pas, de l'implantation perverse, c'est-à-dire d'un domaine contre-nature identifié tel par un pouvoir et enfin de la science sexuelle, qui achève l'analytique interprétative de la sexualité moderne. De la pénitence à la science, «l'homme en Occident est devenu une bête d'aveu ${ }^{12}$,»

Derrière cette formule un peu forte, se tient l'hypothèse la plus fertile du programme de La volonté de savoir. Cette hypothèse concerne la transition de l'époque classique à la modernité, laquelle s'inscrit principalement dans le fait que la vérité du sujet moderne est d'abord le savoir de ce sujet autour de la question du sexe. L'amplification de l'aveu est ordonnée à un régime complexe de savoir, à une vérité du sexe réglée sur le discours qui en est tenu. Aveu de l'examen, aveu de la cure, aveu de la science. Pour rendre cette hypothèse intelligible, ne faut-il pas en premier lieu réintroduire le sujet de cet aveu ? Qui est-il ? Non pas certes le sujet soumis à l'instance d'un pouvoir conçu juridiquement comme loi, interdit, censure. Et pas davantage le sujet de l'analyse, dans son dramatique affrontement au nom du père. Foucault parle plutôt d'un sujet constitué autrement et pour rendre compte de cette constitution différente, il entreprend de structurer un dispositif à plusieurs volets: l'hystérisation du

12. Michel Foucault. La volonté de savoir, p. 80. 
corps de la femme, la pédagogisation du sexe de l'enfant, la socialisation des conduites procréatrices et la psychiatrisation des plaisirs pervers. Ces volets sont en fait des propositions qui se chargent de mettre en forme l'objet historique comme un réseau dans lequel le sujet n'est ni agent, ni victime. Hors de ce réseau, le sujet n'est pas; dans ce réseau, il n'a pas d'importance ${ }^{13}$. Son statut est celui de l'illusion, son nom est le nom d'un effet.

Une telle mise en forme du sujet historique permet peutêtre de comprendre pourquoi la définition fournie par Foucault de la sexualité s'élabore sans concept de sujet. La sexualité, en effet, c'est «l'ensemble des effets produits dans les corps, les comportements, les rapports sociaux par un certain dispositif relevant d'une technologie politique complexe. ${ }^{14}$ »Dans cette définition, la question de la subjectivité n'est pas tant rayée que relayée par un appareil théorique, construit autour des concepts de technique et de dispositif et faisant fond sur une ontologie du corps. Ce n'est pas seulement une question de perspective ou de point de vue; le sujet n'est nulle part posé, et il est partout expliqué, c'est-à-dire produit comme effet dans le corps. La production de la sexualité, par la mise en discours du sexe, appartient au registre de ces effets constitutifs du sujet; elle n'est au demeurant qu'une des formes de la production du sujet moderne.

À s'en tenir à ces formulations, qui sont renforcées par un affrontement quasi systématique avec la psychanalyse, le sujet n'est qu'une outre vide, un reliquat du discours classique dont la référence a cessé d'être celle d'un centre, d'une origine, d'un destin. En se transformant, en passant à la modernité, le sujet herméneutique s'est dissous; toute la profondeur que lui avait donnée autant le christianisme que la psychanalyse, dans la forme unique de l'aveu, s'est aplatie. L'histoire de la sexualité, telle qu'elle est esquissée dans La volonté de savoir, est donc le projet de rendre compte de cette dissolution.

13. Toute cette question du dispositif est discutée dans un entretien avec des analystes de l'Ecole freudienne. Le jeu de Michel Foucault. Ornicar. Bulletin périodique du champ freudien, $n^{\circ} 10$ (1977) 62-93. La discussion est cependant complaisante et n'aborde pas le différend de fond sur les conceprs de la psychanalyse.

14. Michel Foucault. La volonté de savoir, p. 168. 
Mais il y a un autre récit. C'est celui qu'on peut trouver dans un essai rétrospectif, où Foucault reprend la question du sujet par le concept de mode de subjectivation. Dans cet essai, Foucault affirme que le but ultime de son travail n'a pas été de jeter les bases d'une analyse du pouvoir, mais plutôt de produire une histoire "des différents modes de subjectivation de l'être humain dans notre culture ${ }^{15}$. " Il cite d'abord l'objectivation du sujet dans les sciences, par exemple dans la grammaire générale, qui a motivé Les mots et les choses. Il parle ensuite de l'objectivation du sujet dans les pratiques divisantes, qui font de lui un objet, par exemple dans le partage entre l'homme fou et l'homme sain qui fait la matière de L'Histoire de la folie à l'âge classique. Enfin, écrit-il, «j'ai cherché à étudier la manière dont un être humain se transforme en sujet; $j$ 'ai orienté mes recherches vers la sexualité, par exemple la manière dont l'homme a appris à se reconnaître comme sujet d'une sexualité. Ce n'est donc pas le pouvoir, mais le sujet qui constitue le thème général de mes recherches. ${ }^{16}$ 》

Objectivation du sujet, mode de subjectivation: la perspective indique un déplacement. Si le mode de subjectivation doit décrire un procès inscrit historiquement, on doit présupposer que le sujet n'est pas originaire, qu'il peut être analysé dans sa progressive constitution. Dans l'ontologie fragile qui accueille un tel procès, la modalité précède l'être qu'elle travaille à constituer. C'est à structurer cette modalité que tend tout l'appareil de la technologie et du dispositif. On peut se demander, par exemple, s'il y a des dispositifs qui sont plus subjectivants que d'autres? Le dispositif de sexualité est-il de ceux-là et peut-on le périodiser de telle façon qu'on y reconnaisse des étapes distinctes? Foucault propose de distinguer le $19^{\mathrm{e}}$ siècle, comme âge de la découverte du sexe, du $20^{\mathrm{e}}$ siècle, époque de la sexualité à proprement parler, époque du sujet sexuel. Avant, écrit-il, il n'y avait que la chair.

15. Michel Foucault. "Deux essais sur le sujer et le pouvoir. I. Pourquoi étudier le pouvoir : la question du sujet », dans Hubert L. Dreyfus et Paul Rabinow. Michel Foucault. Un parcours philorophique. Au-delà de l'objectivité et de la subjectivité. Traduit de l'anglais par Fabienne Durand-Bogaert. Paris, Gallimard, 1984 (Bibliathèque des sciences humaines); p. 297.

16. Ibid., p. 298. 
Mais qui est la chair? Qui est le sexe? Cette division ne suffit certes pas à faire voir comment la modalité s'introduit pour produire la subjectivation? A lire ce schéma, la subjectivation progresse par plateaux. Elle peut être atteinte épistémologiquement au $18^{\mathrm{e}}$ siècle, mais ne se réaliser sexuellement qu'à l'époque de l'aveu, c'est-à-dire la nôtre. Dans l'évolution des textes de Foucault, «sujet» ne semble donc pas désigner unitairement l'individu, l'être humain, mais un ensemble de fonctions et de statuts par rapport à la connaissance, à l'autre et à la vie sexuelle. L'entreprise de l'histoire est profondément herméneutique, dans la mesure où il lui appartient de thématiser le résultat de l'analyse et de déclarer quand et selon quelle modalité le sujet est constitué. La subjectivation qui est alors atteinte demeure un objet construit et elle est en abîme sur le monde, peut-être seul réel et seul vrai, des réseaux et des dispositifs. Produit dans le discours, le sujet se dispose pour lui-même d'abord dans la croyance; il n'est que ce qu'il croit être. C'est ce qui explique que Foucault puisse maintenir que l'analyse du mode de subjectivation n'a rien à voir avec la vérité ${ }^{17}$.

En déplaçant vers le «mode de subjectivation» la perspective dans laquelle Foucault veut engager rétrospectivement la lecture de son travail, il rend possible ce qui va en devenir une sorte de renversement. Il ouvre ce travail sur un moment de l'histoire, dont il ne cherche à rendre compte par la description d'aucun dispositif, par la mise en forme d'aucune stratégie, par l'efficacité d'aucun pouvoir.

\section{II}

Dans le long intervalle qui sépare le programme de $L a$ volonté de savoir de la publication des recherches sur le monde grec et romain, le sujet, donc, fait retour. Non plus seulement comme effet et fiction à expliquer, mais comme ontologie

17. Ce qui n'exclut pas que l'entreprise soit marquée par une volonté de vérité, comme cela apparaît dans la lecture attentive de Alan SHERIDAN. Michel Foucault. The Will to Truth. London \& New-York, Tavistock Publications 1980. Ce livre est beaucoup plus fidèle à la portée philosophique du travail de Foucault que la synthèse de Dreyfus et Rabinow, peut-être trop soucieuse d'une interprétation compatible avec les sciences sociales d'orientation herméneutique. 
fondamentale du texte éthique ancien. Du mode de subjectivation, on est amené à la considération d'une subjectivité maîtresse, qu'il faut se garder de confondre avec le sujet herméneurique. Il s'agit d'abord d'un sujet éthique, dont les traits se marquent par les objets nouveaux de la généalogie: la mesure des plaisirs, la domination de soi, l'ascétisme et en général, «le souci de soi», epimeleia beautou. Ces objets appartiennent à un temps de l'histoire qui n'est pas celui de la modernité, et ils ne sont pas analysés dans le but d'y conduire: dans la lecture du témoignage ancien de la sexualité, aucun raccrochage à la modernité n'a été conservé, ce qui est peut-être déjà un indice explicatif du reflux de la question du pouvoir. Dit autrement, le retour au sujet est un retour au sujet ancien : le rapport de ce sujet éthique à un sujet moderne n'est pas pensé. La question heideggerrienne d'une conception de l'époque se tient ici en retrait, mais elle n'est pas active.

Foucault avait établi dans La volonté de savoir un processus de constitution réciproque des sciences herméneutiques et du sujet: dans l'aveu, le sujet se découvre, par exemple, sujet de sa folie et accède à une intelligibilité inédite de ce qu'il est en tant que sujet. Au sens propre, il se constitue comme sujet dans l'aveu. Ce premier moment de l'aveu accomplit en quelque sorte le cycle chrétien. Dans un deuxième temps, qu'on peut corréler avec la naissance de la psychanalyse, le sujet établit un rapport avec un autre, de manière à instaurer l'aveu dans l'interprétation et produire la reconnaissance. Cette convergence de l'aveu chrétien dans la confession et du travail analytique a pour résultante un rapport intime et profond de la subjectivité et de l'interprétation au début de notre siècle. Ce rapport n'advient qu'à ce moment-là, il est constitutif.

Le renversement survient quand l'histoire est abordée par l'examen d'une époque où le sujet n'est pas constitué comme sujet herméneutique, mais est pleinement donné comme sujet éthique. Là où Foucault travaillait à montrer comment le dispositif de la sexualité moderne produit un sujet qui lui correspond, il ne peut faire sur le monde grec que l'épreuve d'un sujet souverain et sans origine. Foucault n'en montre aucun processus de constitution, il ne fait qu'en déployer les 
prescriptions et les conséquences. La généalogie est envahie par ce qu'il appelle lui-même une «ontologie historique ».

On peut en distinguer trois types. En premier lieu, « une ontologie historique de nous-mêmes dans nos rapports à la vérité, qui nous permet de nous constituer en sujets de connaissance ${ }^{18}$. "Cette ontologie est au demeurant une forme de la généalogie dont le domaine privilégié est le discours classique, mais rien n'empêche qu'on pourrait en transposer les principes ou la méthode dans une enquête sur le monde ancien. Ensuite, "une ontologie historique de nous-mêmes dans nos rapports à un champ de pouvoir, où nous nous constituons en sujets en train d'agir sur les autres. " Ce deuxième axe motivait l'attirance du programme de La volonté de savoir dans l'orbite de Surveiller et punir, qui en paraissait comme la matrice obligée. Enfin, «une ontologie historique de nos rapports à la morale, qui nous permet de nous constituer en agents éthiques. 》

Cette typologie est floue, mais elle indique assez l'effort de Foucault de séparer une forme de généalogie produite dans la catégorie du pouvoir et une autre qui s'élabore sans cette catégorie. De la même manière, elle indique que si les ontologies développées sur le terrain du monde classique pouvaient tendre à exclure le sujet en le montrant produit, l'ontologie de l'éthique ne peut advenir qu'à partir d'un sujet constituant. L'histoire de la sexualité se trouve donc inversée. Ce n'est plus le dispositif qui produit le sujet, mais le sujet qui commande le dispositif. Tout le matériau grec va en conséquence être travaillé par Foucault selon un modèle où les morales, première et quasi unique source de son enquête, vont présupposer un sujet dont la généalogie n'est pas produite. Le programme de 1976 a été élaboré comme si l'ontologie du pouvoir devait dominer l'entreprise, mais la réalisation s'en avère différente. Déjà les doutes émis sur la validité de ce qu'il appelait l'hypothèse répressive, selon laquelle une histoire de la sexualité est d'emblée une histoire du refoulement, avaient amené Foucault à mettre en question les

18. Michel Foucault. Entretien avec Hubert L. Dreyfus et Paul Rabinow. À propos de la généalogie de l'éthique: un aperçu du travail en cours, dans Hubert $L$. Dreyfus et Paul RABINOW, op. cit., p. 332. L'entretien a eu lieu en avril 1983. 
traits actifs du pouvoir. En introduisant maintenant la «substance éthique», et encore le rapport du sujet à lui-même, qui est bien dans sa division topique le rapport le plus fondamental dans la constitution de la morale, Foucault s'adresse à un sujet qui ne peut plus être une fiction.

L'ouvrage est une trilogie, dont les deux premiers volets sont parus ${ }^{19}$. L'usage des plaisirs est consacré à la période grecque classique, et Le souci de soi prolonge l'analyse à la période hellénistique, grecque et romaine. Le troisième volet sera consacré à la période patristique, sous le titre Les aveux de la chair. Dans les deux morceaux disponibles, le sujet a cessé d'être produit par le discours; il est là, consistant et résistant, inventif et généreux, indépassable souverain de son existence. Le rapport qu'il entretient avec lui-même n'est pas déterminé politiquement et aucun dispositif n'est adonné à décrire une détermination de ce genre. Il faut remarquer d'emblée que cette position correspond à un choix de Foucault, puisque plusieurs aspects de la vie sexuelle ancienne semblent avoir été absolument déterminés politiquement. C'est le cas, par exemple, de l'homosexualité. En adoptant une méthode qui privilégie le discours moral sur ses éventuelles déterminations, Foucault établit une stratification inattendue de l'ontologie. L'histoire de la sexualité passe en effet ici par l'analyse d'un discours qui la mesure et la règle, sans que soient mises en question les assises de ce discours. Cette attitude laisse une place royale au sujet éthique et marginalise la considération du politique.

Pour évaluer le parcours qui a conduit à faire cette lecture particulière de la sexualité antique, il est important de souligner la réintroduction dans l'ouvrage d'un concept d'expérience. L'histoire de la sexualité comme histoire de l'expérience n'est pas une histoire empirique, encore que l'empiricité de ses objets doive y contribuer. Par l'expérience, Foucault veut engager toutes les correlations à l'œuvre dans une culture entre les domaines de savoir, les types de normativité et les formes de subjectivité. La réintroduction de la catégorie du sujet entraîne donc une réappropriation d'un concept d'expérience très vaste. L'accès à

19. Michel Foucault. L'usage des plaisirs et Le souci de soi. Paris, Gailimard, 1984 (Bibliothèque des Histoires); 285 et 284pp. 
l'expérience est d'emblée sédimenté. Dans le cas de l'éthique, on se trouve confronté à plusieurs possibilités: s'agira-t-il de faire une histoire des codes moraux, des prescriptions explicites, des règles, des lois, ou alors et autrement, des pratiques, habitudes, comportements réels? La réponse de Foucault est qu'aucune de ces approches n'est la plus riche. Le sujet n'existe pour ainsi dire que dans l'écart entre la prescription et le fait. Il consiste précisément dans cette relation à soi, dans ce rapport qui détermine comment l'action est moralement assujettie. Plus largement, comment l'existence intègre la substance éthique. L'expérience est donc une tension; ce n'est pas un fait, qui demeurerait inaccessible, mais un rapport au fait.

Une remarque s'impose sur ce préalable. Ce concept de sujet n'est pas indifférent, ce n'est pas n'importe quel sujet, mais un sujet philosophique, surtout platonicien, divisé et en lutte dans sa division, toujours déjà inscrit dans le rapport conflictuel du désir et de la raison. Le premier effet de la rencontre de Foucault avec le monde grec est précisément la prise en charge de ce clivage. Dans tous ses aspects, puisqu'il ne fera l'économie d'aucune analyse pour retracer et exprimer ce rapport de soi à soi partout où on peut le retrouver. Bien entendu, cette enquête se limite aux aspects conséquents de la sexualité, et elle ne s'étend pas à toute l'ontologie du sujet. Mais chaque fois que la sexualité permet d'éclairer ce rapport de soi à soi, cette distance topique, Foucault y trouve plus de signification qu'autrement. Les domaines de la sexualité qui ouvrent ce rapport sont aussi bien ceux qui l'engagent vers le conflit et la topique platonicienne qui domine, à partir de la République et du Pbèdre, toute la philosophie grecque, en constitue le modèle privilégié. Une lecture élaborée à partir de textes plus populaires aurait sans doute donné d'autres résultats, mais Foucault a choisi d'interpréter la sexualité d'abord dans la philosophie. C'est un choix qui le renvoie au Banquet, dont il fournit une analyse très riche et dont il fait le pivot de toute l'expérience grecque de la sexualité. Moralisée dans une topique du conflit, la sexualité n'en constitue pas moins la porte d'entrée dans la vérité. Elle se révèle ainsi isomorphe à la philosophie, qui est à la fois ascèse et transcendance.

Dans un texte important, placé au début des ouvrages que nous examinons, Foucault a proposé de voir quatre aspects 
fondamentaux du rapport à soi. C'est d'abord la part du sujet qui se trouve en rapport avec une conduite morale. Cette part peut varier.

En acceptant de parler de "part» de l'individu, ce n'est pas tant la topique dans ses instances qui est convoquée que la manière même dont celle-ci prend une forme substantielle. Si l'individu donne préséance au respect du code, il constitue une certaine part de lui-même comme substance éthique. Dans les termes de la psychanalyse, que Foucault évite ici soigneusement, on dirait qu'il adopte une position surmoïque. Peut-il faire autrement? La variation possible, indiquée dans le texte, ouvre à la fois sur la maîtrise du désir et sur le contenu de l'expérience. Une telle ouverture, si elle était davantage explicitée, livrerait la conceptualité du sujet qui est à l'œuvre dans tout le travail, et qui lutte pour dépasser le surmoi freudien. Il n'est pas certain qu'elle y parvienne: la substance éthique, ce ne peut être que le sujet se déterminant lui-même dans le langage, dans la contradiction avec l'expérience. Chercher à la déplacer du respect de l'interdit vers l'ascèse ou encore vers le contenu intrinsèque de l'expérience modifie certes la morale: on passe alors d'une morale formelle, hétéronome, à une spiritualité et éventuellement à une forme classique d'eudémonisme. Mais dans ces trois paradigmes de la substance éthique, - respect, domination, jouissance - c'est encore un même sujet qui préside, à proportion que c'est le sujet qui s'y dispose. Foucault manque ici une occasion importante de préciser, à l'intérieur même de l'éthique, les statuts différenciés de la subjectivité. Le sujet prend forme et se manifeste différemment, selon qu'il investit la pratique morale dans le souci d'un rapport avec la Loi, dans la volonté d'être lui-même un souverain moral ou enfin d'identifier son bien et son bonheur.

$\grave{A}$ ce premier foyer de différences, Foucault juxtapose aussitôt quelques exemples qui permettent d'en mesurer la portée. La substance éthique moderne présuppose un sujet volontaire, intentionnel; le surmoi est en ce sens une entité profondément kantienne, dont rien d'équivalent ne peut être retrouvé dans la culture grecque qui ne moralise pas tant la Loi que les actes. Foucault isole à cet effet les aphrodisia, c'est-à-dire les actes liés dans leur unité au plaisir et au désir. Ce n'est donc ni la chair chrétienne, ni la sexualité moderne. Le sujet ancien est 
dans un rapport d'usage (chrēsis) avec les aphrodisia, et en retour ce rapport l'écarte aussi bien de la Loi que de la jouissance. Une telle caractérisation laisse donc supposer que d'une époque à l'autre la substance éthique se modifie; elle engage à ce titre une interprétation spécifique de la culture grecque et le modèle exemplaire de son sujet.

Cette proposition est tout à fait problématique, dans la mesure où Foucault en cherchera la vérification non pas dans l'archive ou le témoignage de la vie morale historique, mais dans les morales prescriptives, surtout stoïciennes. La détermination de la substance éthique, telle qu'il la présente trop imprécisément est sans commune mesure avec la constitution de l'éthique philosophique. L'éthique préconise le critère du jugement moral, elle en décrit le fondement; par elle-même elle est indifférente au sujet auquel elle s'adresse, et se trouve donc toujours confondue avec la Loi. La substance éthique dont parle Foucault fait l'économie de l'éthique spéculative, qu'elle exploite paradoxalement comme texte de culture. Ce glissement n'est pas justifié. Déterminer la substance éthique c'est en quelque sorte déterminer le terrain sur lequel une époque accepte la prise de l'éthique. Les effets théoriques que Foucault semble escompter de cette proposition sont les suivants: elle rendra possible une caractérisation, elle sera vérifiable dans le texte éthique. Cette méthode ne serait susceptible d'aucune circularité si on pouvait montrer que l'histoire, - entendons par là la communauté des sujets historiques - , choisit dans le texte éthique les formes dans lesquelles elle acceptera de manifester son modèle de sujet. Cela suppose une confrontation des morales écrites et des philosophies avec les choix des individus. Chez Foucault, cette entreprise n'a pas lieu, et le texte éthique de la culture grecque retourne à luimême, laissant dans l'ombre le sujet supposé se déterminer contre lui et qui s'avère finalement déterminé par lui. Le résultat est qu'aucune détermination particulière n'apparaît ici qui ne soit le fait du texte éthique.

Il y a donc dans cette présentation de la méthode un espace flou concernant les différents aspects du sujet appelés à se constituer moralement, et pour qu'il fût de contour plus net, il aurait fallu faire appel à la représentation du sujet non seulement 
dans l'éthique, mais partout ailleurs où il a laissé sa trace, et notamment dans la mythologie et la tragédie.

Pour compléter le travail des différences, Foucault introduit ensuite le mode d'assujettissement, savoir le rapport à la règle, le mode selon lequel les individus ont à reconnaître les obligations morales qui s'imposent à eux. Cet aspect de la subjectivation concerne les fondements éthiques que chacun accepte de reconnaître pour vrais, la nature, le Dieu qui parle dans la Révélation, la loi humaine. Le sujet s'éprouve donc dans un rapport particulier à une instance du Bien, qui peut en contredire d'autres et qu'il a pouvoir et privilège de choisir. Si la détermination de la substance éthique est le mode de la moralité, sa prise ontologique, l'assujettissement en est comme la raison, la justification explicite en même temps que la croyance et l'engagement. Ce niveau de la morale est impossible à atteindre par la seule considération du texte. Foucault lui confère la même amplitude, la même ouverture que la substance éthique: conformité, responsabilité, perfection répètent ici les possibilités de soumission, de domination et de jouissance de la substance éthique. Dans ce tableau redoublé des possibilités du sujet se confirme le caractère traditionnel, hérité de la topique ancienne, de son modèle: le sujet hésite entre la servitude, la maitrise reposant sur le conflit (dans l'ascèse et la tradition spirituelle), et enfin la fusion dans l'existence et la jouissance, où semblent disparaître non seulement l'altérité de la Loi, mais le conflit même du désir et de la raison.

Le troisième aspect de cette dialectique du sujet est le travail opéré sur soi-même, qu'on peut appeler aussi travail éthique. «Sculpte sans cesse ta propre statue », écrivait Plotin. Sous cet impératif se condensent bien des finalités spirituelles, et en particulier toutes les variétés de l'ascèse, jusqu'aux conduites limites de l'abstinence sexuelle et de la virginité. L'antiquité grecque a été extrêmement riche en exemples et en propositions philosophiques à cet égard. L'ensemble de la philosophie ancienne, même spéculative, même scientifique, se laisse réinterpréter comme un exercice spirituel dont la domination de soi n'est que le premier moment et la contemplation l'étape 
ultime. Ne voit-on pas, chez Plotin, le travail éthique apparaître comme le seuil même de la vie philosophique? 20

À cet aspect proprement spirituel et ascétique, Foucault en joint aussitôt un dernier : la téléologie du sujet moral, c'est-à-dire cet aspect de la subjectivité qui règle une conduite morale sur une fin plus haute, plus englobante. On rencontre ici le rapport du sujet à la totalité de l'action morale, par rapport à laquelle il tente de se définir comme valeur et ultimement peut-être comme style. "Quelle sorte d'être voulons-nous devenir lorsque nous avons un comportement moral? "

L'ensemble de ces modes de subjectivation constitue un effort sans précédent chez Foucault pour penser l'articulation du sujet et de la loi. La structuration qu'il propose passe par une intériorisation de la substance éthique qui déplace considérablement l'espace d'analyse des morales et des comportements. Le soi moral est établi d'emblée dans la forme du sujet, essentiellement dans la modalité de l'écart, de la division et en conséquence de la domination. Ce modèle renvoie dans la marge une analyse politique, pivotant sur le concept du pouvoir.

C'est aussi l'établissement de ce modèle du sujet qui rend possible la superposition d'une histoire de la sexualité et d'une histoire de la morale. Le matériel qui se trouve d'abord exploité consiste en discours, lus pour leur valeur d'injonction et leur exemplarité : les formes de la subjectivité structurent à la fois les domaines de savoir et les types de normativité. Précisons que cette morale est à la fois médicale et philosophique, puisque les témoignages sollicités présupposent une sorte d'interpénétration

20. C'est aussi le sens de la structuration propédeutique du corpus platonicien dans l'Académie, qui plaçait l'Alcibiade comme porte d'entrée dans la philosophie. Voit sur ce sujet le livre de Jean PÉPIN. Idées grecques sur l'bomme et sur Dieu. Paris, Les Belles-Lettres, 1971. Dans sa leçon inaugurale au Collège de France, où il occupe depuis 1983 la Chaire d'histoire de la pensée hellénistique et romaine, Pierre Hadot insistait sur la connaturalité en Grèce de l'exercice de "raison» et de la méditation. Dans son livre Exercices spirituels et philosophie antique (Paris, Études Augustiniennes, 1981), on trouvera plusieurs analyses qui conduisent à la même conclusion: le travail philosophique est d'essence spirituelle. La parenté des travaux de Foucault avec ceux de Pierre Hador à la même période ne me semble pas une pure coïncidence ; pour avoir suivi leur enseignement au Collège de France au cours d'une même année, je puis attester d'une extraordinaire communauté d'intérêt. 
de la médecine et de la philosophie, dont la distinction dans l'Antiquité à bien des égards était moins forte qu'à d'autres époques. Le matériel littéraire n'est pas utilisé, encore moins l'iconographie ${ }^{21}$. La subjectivation est donc inscrite d'abord dans la philosophie, dans une philosophie qui intègre naturellement l'étude du corps, des humeurs, des diètes. La lecture du Timée de Platon et du Corpus de Galien montre que cette relation de la médecine et de la philosophie n'a pas beaucoup varié dans l'Antiquité.

Dans ce matériel complexe, où la même prescription règle à la fois la morale et la santé, le sujet se trouve d'abord déterminé par la formule de son rapport à lui-même. De l'usage des plaisirs au souci de soi, la formule a varié: le monde hellénistique se révèle plus préoccupé de refermer le sujet sur lui-même, là où le sujet classique pensait encore se poser dans une domination réglée des plaisirs et en général du monde. Mais dans les deux cas, le sujet est originaire, déjà constitué dans son rapport à la sexualité. Les modes de subjectivation proposés par Foucault s'appliquent aussi bien, en effet, à l'usage des plaisirs qu'au souci de soi, et ils permettent un modèle qui manifeste le déplacement de l'objet.

Cette représentation du sujet est-elle juste? En acceptant qu'une histoire de la sexualité soit par essence une histoire de la philosophie, Foucault présuppose peut-être cela même qui était l'enjeu le plus crucial de son entreprise : que le sujer sexuel est d'emblée moral, toujours déjà moral. Cette question: la sexualité était-elle d'emblée dans l'antiquité domaine moralisé, domaine de l'éthique?, est-il acceptable de ne pas l'ouvrir dès le point de départ, en ne supposant pas constitué le sujet dont il s'agit de montrer la progressive formation? Foucault semble avoir voulu précisément éviter une sorte d'histoire téléologique, organisée et commandée à partir du sujet chrétien: ce n'est pas en tant que

21. Il existe, bien entendu, beaucoup de travaux voués à produire une histoire documentaire ou littéraire de la sexualité grecque er romaine. On pense par exemple au livre de K.D. Dover. Greek Homosexulity. London, Duckworth, 1978, qui a renouvelé l'étude de l'homosexualité en exploitant le témoignage de la peinture sur vases. FoucAult ne semble pas avoir utilisé un livre aussi bon que celui de Hans LICHT Sexual Life in Ancient Greece. London, Routledge, 1932. Son intérêt s'est porté quasi exclusivement sur le texte prescriptif, et non sur l'archive. 
pensée de la subjectivité que le christianisme infléchit une histoire de la sexualité, mais en tant que proposition d'un sujet herméneutique, d'un sujet de l'aveu (et peut-être fondamentalement, de la culpabilité). L'identification du sujet moral et du sujet sexuel, qu'on aurait attendue au terme, paraît donc placée délibérément au rang de postulat.

Cette proposition rend l'ensemble de l'entreprise difficile à interpréter. Foucault parle, au sujet de son œuvre, de la nécessité de produire la généalogie du sujet de désir, de faire l'histoire de l'auto-reconnaissance de ce sujet comme sujet de désir. Le désir apparaît comme le fond de l'être, il est la vérité de l'être, naturel ou déchu, qui s'avoue obstinément tel dans la modernité. Comment comprendre, dans les modes de subjectivation qui forment le nouvel appareil de l'histoire de la sexualité, que le sujet de désir soit devenu sujet moral ? Foucault procède donc à grandes enjambées. D'un dispositif qui fait l'économie du sujet à la généalogie du sujet de désir, et puis encore du sujet de désir au sujet moral originaire, le retour du sujet semble l'effet d'un télescopage inattendu.

La question qui vient ensuite n'a de réponse que dans les fondements de la philosophie ancienne. Il était certes difficile pour Foucault de la soulever sans engager toute la métaphysique grecque. Cette question est la suivante : pourquoi le comportement sexuel, les activités et les plaisirs, font-ils l'objet d'une préoccupation, d'un souci dont la dynamique morale va se retourner sur le sujet lui-même à la fin de l'antiquité ?

Dit autrement, pourquoi assistons-nous à une problématisation morale de la sexualité, dès lors qu'elle se thématise comme comportement? La réponse de Foucault, qui présuppose, comme on vient de le dire, un sujet moral, fait appel à la détermination de ce sujet comme sujet de souci. Epimeleia. La problématisation morale, en effet, est liée aux arts de l'existence aux techniques de soi, véritables esthétiques de la vie personnelle qui perdront toute leur importance avec la pastorale chrétienne. La raison de l'éthique apparaît, en son point ultime, fonction d'une esthétique du sujet.

L'insistance sur le souci est certainement une des intuitions les plus riches de Foucault dans ces deux livres. L'epimeleia ne 
caractérise pas seulement la philosophie tardive, mais elle connote déjà chez Platon les activités de l'âme qui sont liées à l'investissement du désir, à la topique. Rien ne semble plus fondamental chez Platon que l'expérience de la division et le souci qui en dérive. Rien ne semble plus ultime non plus: la réinterprétation du mythe de l'androgyne primitif par l'exercice philosophique de la contemplation, du retour à la patrie originaire de l'unité avec les Formes intelligibles, rend impossible une philosophie du désir. Le sujet ne peut que chercher à traverser son désir pour le transformer en philosophie, il ne peut en rendre compte.

La représentation topique du sujet impose donc un art de la réflexivité: il s'agit toujours de se connaître, de se faire, de s'éprouver, de se maîtriser pour ultimement se retrouver. Cette réflexivité est indépassable, aussi longtemps qu'est maintenue une hiérarchie de fonctions, dans laquelle une raison directrice commande notamment au désir. L'epimeleia platonicienne marche de pair avec l'bégémonie, dont les stoïciens feront le concept même de l'âme. La problématisation morale chez Foucault renvoie donc ultimement à une structure du sujet. L'usage, aussi bien que le souci, n'ont de sens que sur le fond de ce sujet divisé, pensable uniquement dans la figure du maître ou du commandant (bēgemōn).

On atteint ici une sédimentation du texte philosophique ancien qui permet peut-être de saisir le préalable ontologique de l'éthique. Ce sujet structuré n'est lui-même tel, en effet, que parce qu'il a été conçu dès Platon, et sans qu'Aristote modifie suffisamment la conception de son maître pour que son éthique s'en trouve altérée, comme un dualisme. Chez Platon, ce dualisme ne saurait être limité à une conception du sujet, mais il est développé dans une métaphysique qui sépare un monde transcendantal de Formes, dont l'âme est parente dans son incorporéité, d'un monde matériel qui n'en est que le reflet. Toute la philosophie grecque, jusqu'au stoïcisme romain, peut être représentée comme un effort pour rendre compatible la métaphysique platonicienne avec une conception non dualiste du sujet.

Pour ce sujet divisé, ontologiquement double, la sexualité n'est pas le premier, ni même le plus important des domaines de 
l'existence auxquels peut s'appliquer le travail éthique. Dans la tension du corps et de l'âme, et dans les conflits intérieurs de l'âme, ni Platon, ni Aristote, ni la philosophie tardive ne lui ont accordé une place prépondérante. Le concept des "passions», notamment, ne peut être interprété dans un sens exclusivement ou même principalement sexuel. Il recoupe plutôt l'ensemble de la vie du corps, et ce qui par le moyen du corps affecte les parties inférieures de l'âme. Cela, Foucault est contraint de le reconnaître en renonçant à un domaine spécifique du sexuel, au profit du domaine plus large des apbrodisia, comme substance éthique de la Grèce classique. Ce n'est donc pas tant la sexualité qui se trouve moralisée que l'ensemble de l'activité de plaisir. À cet égard, les actes et les comportements sexuels revêtent en Grèce une certaine banalité ; ce qui compte, c'est l'usage mesuré des plaisirs, l'équilibre des investissements. L'hégémonie de l'intellect, qui est le fondement ontologique de toute l'éthique grecque, conduit au projet de régler toutes les passions. Dominer et régler ont ici un sens très voisin, dans une économie du désir qui est à la fois usage tempéré et sublimation dans la connaissance et la philosophie.

La problématisation morale de Foucault, toute sa réflexion sur les modes de subjectivation n'est donc pas si aporétique; elle prend tout son sens sur le fond du dualisme métaphysique platonicien qui traverse toute la pensée ancienne. C'est à partir de lui qu'on pourrait tenter de remonter à des origines pythagoriciennes du sujet divisé, et c'est là aussi qu'on retrouverait les formulations les plus primitives du lien entre la médecine, la diététique, l'ascétique et l'exercice de la philosophie. Foucault a certes raison d'évoquer pour rendre raison de cette préoccupation constante de l'usage et de la mesure, le principe même du précepte esthétique ou stylistique qui va marquer l'éthique tardive du souci de soi : ce thème est présent, sensible, mais il n'est que la manifestation d'une ontologie plus profonde du sujet ancien, ontologie de la coupure et de la domination.

Si donc on doit chercher à interpréter les thématisations anciennes de l'usage des plaisirs et du souci de soi, ce ne peut être simplement selon un modèle où la morale se confond à une esthétique. On doit faire réintervenir le concept ancien du sujet, l'âme, qui du Banquet de Platon au traité sur l'Eros de Plotin (Enn., III,S), lie métaphysiquement le sujet maître immatériel à 
son existence de désir et de passion. En isolant les thèmes de l'austérité sexuelle, qui révèlent selon Foucault une opposition du comportement de réserve et la crainte de la dépense stérile, les thèmes de l'institution du mariage, de l'homosexualité masculine et encore de l'abstinence en vue d'une existence de sagesse, on ne peut prétendre aborder des problèmes isomorphes. L'austérité et l'abstinence sont des modes de subjectivation spirituels, qui peuvent trouver un fondement dans une métaphysique. On y décèle un rapport très particulier de l'usage des plaisirs à la jouissance, laquelle s'abolit dans la sublimation. En revanche, le mariage et l'homosexualité sont des pratiques et ultimement des rituels, dont le code renvoie à une anthropologie. L'explication de la domination que fournit Foucault au sujet de l'homosexualité ne requiert en effet aucun passage par la métaphysique de l'âme. Le rapport à la philosophie et à la constitution de la subjectivité se trouve, dans son interprétation, moins direct, plus oblique. En refermant cette interprétation sur le concept de «stylisation de l'activité», Foucault assigne au sujet l'espace même de sa maîtrise. Mais il ne lui permet aucune ouverture sur la jouissance, qui se trouve en quelque sorte la polarité positive extatique de l'usage.

L'usage ne renvoie-t-il pas au plaisir comme accomplissement, la chresis n'est-elle pas pensée en fonction de l'bēdonêe? L'uti en vue du frui. Foucault se garde bien de travailler de front cette question de la jouissance et du plaisir, laquelle marquerait sans doute un lien trop moderne, peut-être freudien d'essence, entre le plaisir et la sexualité. Cette attitude se marque notamment, dans son entreprise, à une attention quasi exclusive au stoïcisme, au détriment d'une pensée du plaisir épicurienne, laquelle aurait sans doute modifié la perspective d'ensemble. C'est, en fait, en vertu du même refoulement que l'épicurisme et la pensée du plaisir/jouissance sont écartés de cette histoire de la sexualité, où le sujet est d'abord domination, mesure, souci.

De ce point de vue, l'appareil à quatre thèmes apparaît donc comme une construction assez fragile. Ces quatre types de stylisation de la conduite sexuelle, que Foucault résume ainsi: diététique du corps, économique du mariage, érotique des garçons et philosophie de la vérité, ne sont pas des ensembles bien formés. Chaque type joue sur les autres, et c'est la formule 
générale qui semble conduire à l'idée que ces types font système, savoir qu'il est possible de mettre au jour quatre domaines où les aphrodisia étaient l'objet d'une pratique stylisée. La question qu'il faudrait pouvoir retourner à Foucault devient peut-être la suivante: est-ce bien la chrēsis qu'il fallait interroger pour tenter de structurer la sexualité ancienne? Tout n'est-il pas donné déjà dans cette notion même de $c h r \bar{e} s i s$, dans ce concept de mesure et d'usage qui enferme le sujet dans la figure de la maîtrise et de l'écartement ? Ce sujet, toujours déjà à distance de son désir et de son plaisir, est-ce bien lui qui peut guider une histoire de la sexualité ? N'est-il pas déjà le sage, le philosophos, qui conjugue en même temps esthétique et morale, stylistique et ascétique, hors du chemin pavé à la sexualité ordinaire et à son discours propre.

Dit autrement, en ayant recours à ce sujet distant, et peutêtre artificiellement construit sur la base de quatre domaines d'activité finalement très hétérogènes, Foucault pose un sujet plus que moral, un sujet ascète et idéalement abstinent. Ce choix du terrain de la philosophie impose une sélection de sources en même temps qu'une interprétation et des modèles.

La question peut être retournée d'une autre façon, et nous retrouverons les formulations qui ont animé notre lecture depuis le début. Est-ce le fait de recourir à la philosophie qui a amené Foucault à réinvestir le concept de sujet dont le programme de toute son entreprise depuis 1976 prévoyait se passer? N'aurait-il pas été pensable de conduire le projet de La volonté de savoir dans le domaine d'une histoire de la sexualité non déjà retravaillée par le souci spirituel de la philosophie? C'est une question qui mérite d'être posée. Y a-t-il en Grèce, pour ce projet, autre chose que la philosophie? ${ }^{22}$

22. Beaucoup de travaux, outre celui de Hans Licht cité plus haut, le montreraient avec abondance. Par exemple, le travail de Sarah B. Pomeroy, Goddesses, Whore, Wives and Slaves: Women in Classical Antiquity. Berkeley, 1975. Et encore, Bernard SeRgent. L'bomosexualité dans la mythologie grecque. Paris, Payor, 1984. Voir enfin Aline Rousselle. Porneia Paris, P.U.F., 1984. Dans la philosophie, d'autres pratiques d'interprétation sont possibles; par exemple, J.C.B. GosLING \& C.C.W. TAYLOR. The Greeks on Pleasure. Oxford, 1985 et Florence Dupont. Le plaisir et la loi. Du Banquet de Platon au Satiricon. Paris, F. Maspero, 1977. 
La réponse à cette question se trouve dans le choix de départ de Foucault: tenter de saisir comment le sujet s'est constitué comme objet de savoir pour lui-même, dans la modernité. En déplaçant son enquête vers l'Antiquité, Foucault s'est trouvé moins préoccupé de la question de l'aveu, thème fondateur de la constitution du sujet herméneutique par le christianisme et par la psychanalyse. Il a pu retraverser toute l'épaisseur de la tradition chrétienne et retrouver derrière l'aveu et le moi des profondeurs, un autre sujet, plus originaire, le sujet éthique. Ce n'est pas la prolifération de l'aveu qui le constitue, ni les techniques de l'interprétation, mais le souci. On entend ici résonner le sorgen heideggerien derrière l'epimeleia grecque, mais le langage qui recueille ce souci participe autant de la morale et de la politique que de l'ontologie. Chez Platon, ce sujet est un sujet de maîtrise et l'epimeleia, que Foucault a plaisir à contraster avec la chrēsis, est liée d'ores et déjà à la mathessis, à la didaskē, à l'askēsis. (Protagoras,323d8) Les Stoïciens, penseurs habités par le langage de la maîtrise, ne cesseront de retravailler le concept de la psychè par les figures du gouvernement et de la souveraineté. Contrairement au sujet moderne, on ne peut tenter de dissoudre l'âme ancienne dans des procédures d'objectivation; derrière la philosophie demeure le mythe, qui garantit au sujet son origine, sa légitimité, tout en racontant dans les péripéties de la métaphysique les causes de son destin et de sa tragique division.

Foucault a rendu compte d'au moins un aspect important de ce sujet ancien en insistant sur le fait que ce n'est pas l'interdit (par exemple, la prohibition de l'adultère) qui a amené la réflexion éthique la plus riche, mais au contraire les domaines où la liberté était la plus grande, comme l'érotique des garçons, ou la recherche de la vérité. C'est dans ces domaines, en effet, que le motif de la pratique de soi, de la maîtrise, pouvait et devait être le plus déterminé. Là où la dépense sexuelle était non circonscrite par l'institution, la retenue, l'usage mesuré, même hors de la visée de la jouissance, devint constitutive, éthique, subjectivante Cette observation a pour effet de mettre en relief comment le travail philosophique ancien s'est rigorisé dans ces domaines qui n'étaient pas ceux des interdits et des prohibitions, contrairement en cela, peut-être, à la période moderne, plus soucieuse de légitimation de l'institution. De ce point de vue, Michel Foucault 
se trouve à atténuer passablement le passage du monde grec au monde chrétien, souvent présenté comme un passage de la liberté et de la dépense à l'austérité et à la répression. Sans doute cette opposition ne tient-elle pas. Toute la philosophie grecque postule un sujet austère, adonné à la philosophie comme à une ascèse, c'est-à-dire littéralement à un exercice. Le thème de cet exercice peut se déplacer, sa rationalité peut se mobiliser pour des objets différents, - dans l'angoisse et la peur chez Epicure, dans l'extase chez Plotin mais toujours le sujet grec demeure le sujet du souci.

Je crois, comme j'ai essayé de le montrer, que Foucault a tort de restreindre l'interprétation de ce souci, et de tous les arts de l'existence qui le connotent, à la seule esthétique de la vie, à la pratique d'un style. Peut-être, dans le stoïcisme romain, qui a perdu malgré les efforts de Cicéron, son contact avec les métaphysiques classiques dont dérivaient ces pratiques, trouve-t-on plusieurs figures de ces philosophes esthètes. Mais le stoïcisme, comme Hegel l'a profondément saisi, est une pensée qui renonce au temps, à l'histoire; elle renonce aux péripéties fondatrices du sujet ancien. Partout ailleurs, ces pratiques de stylisation sont traversées par une représentation agonique du sujet, à la fois maître et esclave de lui-même, qui s'articule de manière ultime sur un mythe d'origine. Dans ces mythes, la puissance à vaincre est ontologiquement étrangère, c'est un monde dégradé dans lequel le sujet a chuté. Dans les métaphysiques qui les reprennent, cette puissance a conquis l'existence, elle arrive à s'y identifier: c'est l'action aristotélicienne qui fait échec à la contemplation, ce sont les troubles et les passions de l'âme chez les Stoïciens.

"L'homme le plus royal, écrivait Platon, est roi de luimême.» (République, IX, 580c) Cette idée de royauté, la philosophie grecque n'a cessé de l'explorer, aux fins mêmes d'isoler le principe de ce que nous appelons le sujet et pour lequel les grecs n'avaient pas de nom. Ce qui a son principe en soi-même, ce qui est automoteur, ce qui est selon soi, par soi, ce qui est souverain, ce qui ne dépend que de soi : périphrases et métaphores du sujet ancien. La grande différence, me semble-t-il, et ce point Foucault ne l'aborde pas, entre le sujet ancien et le sujet moderne ou même chrétien, c'est que le sujet chrétien est solitaire, alors que l'âme 
grecque avait partout, dans l'univers visible et invisible, des parents, des démons, des génies et jusqu'à ces âmes du monde et des astres, que la philosophie représente comme connaturelles. Le sujet ancien ne possédait pas le destin de l'individu établi dans un face à face avec un dieu personnel, disposé à son endroit à la fois comme son origine et comme le garant de son intériorité. Un dieu plus intime au sujet, selon la parole de saint Augustin, que le sujet lui-même. En établissant sa royauté sur lui-même, le sujet platonicien n'a en vue aucun modèle royal, aucun Seigneur et aucun Maître autre que lui-même, mais la seule royauté abstraite et splendide de tout ce qui dans l'être est aussi roi de soi-même et prince de soi. Peut-être est-ce en raison de cette existence dépourvue d'un Seigneur, c'est-à-dire d'un rapport à un autre, que Foucault évoque pour le sujet grec le seul concept de l'esthétique. Dans la solitude, le bien s'excède et s'abolit dans la beauté.

Je termine ces réflexions en revenant sur la schématisation d'ensemble que Foucault propose à une histoire de la sexualité qui serait en même temps une histoire du sujet. Pour lui, le christianisme a repris à son compte les techniques de soi, il les a prolongées, mais en même temps il a opéré une rupture assez radicale, dont nous savons, même si le troisième livre n'est pas encore publié, que Foucault l'impute d'abord à Tertullien. Il le compare en importance à Freud. Son rôle aurait été d'avoir brisé le rapport du désir et du plaisir et d'avoir insisté sur les dangers propres au désir, là où les grecs n'avaient souci que de la mesure des plaisirs. De la maîtrise de soi, on passe donc à la purification du désir lui-même, à une ascèse de l'intention dans la suspension de l'acte même. La perspective de la jouissance se trouve doublement décalée, reportée, interdite: prohibée dans la réalité, prohibée dans le désir.

Dans les termes de Foucault, ce mouvement équivaut à transformer le souci grec de soi en une recherche de la vérité du soi dans l'examen, dans l'interprétation et ultimement dans la pastorale de la confession. Il faudra attendre la démonstration de cette proposition dans les aveux de la chair, pour mesurer si certe différence est importante pour séparer le sujet chrétien du sujet ancien. Cette différence a déjà fait l'objet de plusieurs interprétations profondes, notamment chez Heidegger. En général, ces hypothèses ont cherché à isoler ce que le 
christianisme a donné au sujet pour le constituer, la conscience, le sentiment de la faute, la culpabilité, mais aussi la charité, le rapport à l'Autre. Mais au fur et à mesure qu'on progresse vers une interprétation ouverte, - et à ce travail Foucault a beaucoup contribué -, on ne peut plus se contenter de nier platement le sujet ancien, de le banaliser chez Homère, de le vider en rendant sa substance aux forces occultes de la tragédie, d'être aveugle à la topique ancienne de l'âme, de barrer le sujet partout où on ne trouve pas l'aveu. Car d'ores et déjà, la philosophie ancienne a posé ce sujet divisé, sujet de la chrēsis, sujet de l'epimeleia, et même si Foucault n'est pas remonté comme tout l'y invitait de la morale à la métaphysique de l'âme, il a trouvé ce sujet sur son chemin. En travers, et pleinement consistant.

Et comme ces bâtiments chrétiens, que notre langue a choisi d'appeler églises, rendant ainsi à la communauté de l'ekklēsia un privilège sur la solitude royale de la basileia ancienne qui se trouve construite dessous, on ne peut que travailler encore et plus à redécouvrir derrière le sujet soi-disant primitivement chrétien la figure éthique et royale du sujet grec.

Département de Philosophie

Université du Québec à Montréal 